\title{
Common random fixed point theorems for contractions of rational type in ordered metric spaces
}

\author{
Sukh Raj Singh ${ }^{1, *}$, R D Daheriya ${ }^{1}$, ManojUghade ${ }^{2}$ \\ ${ }^{1}$ Department of Mathematics, J. H. Government P. G. College, Betul, India-460001 \\ ${ }^{2}$ Department of Mathematics, Sarvepalli Radhakrishnan University, Bhopal, India-460001 \\ *Corresponding authorE-mail:srsinghssm@gmail.com
}

\begin{abstract}
In this paper, we prove some common random fixed point theorems for mappings involving rational expression in the framework of metric spaces endowed with a partial order using a class of pairs of functions satisfying certain assumptions.
\end{abstract}

Keywords: Altering Distance Function; Contractions; Random Fixed Point; Partially Ordered Set; Metric Space.

\section{Introduction}

Random nonlinear analysis is an important mathematical discipline which is mainly concerned with the study of random nonlinear operators and their properties and is much needed for the study of various classes of random equations. Of course famously random methods have revolutionized the financial markets. Random fixed point theorems for random contraction mappings on separable complete metric spaces were first proved by Spacek [26] and Hans [8-9]. Random fixed point theorems for contraction mappings on separable complete metric spaces have been proved by several authors (Chang and Huang [5], Huang [11], Itoh [12], Liu [16], Papageorgiou [18-19] Shahzad and Latif [25], Tan et al. [27]). The stochastic version of the well-known Schauder's fixed point theorem was proved by Sehgal and Singh [24].

The aim of this paper is to establish some random common fixed point theorems for mappings involving rational expression in the framework of metric spaces endowed with a partial order using a class of pairs of functions satisfying certain assumptions.

\section{Mathematical preliminaries}

The following preliminaries chosen from [13].

Let $\left(X, \beta_{X}\right)$ be a separable Banach space, where $\beta_{X}$ is a $\sigma$-algebra of Borel subsets of $X$, and let $(\Omega, \beta, \mu)$ denote a complete probability measure space with measure $\mu$ and $\beta$ be a $\sigma$-algebra of subsets of $\Omega$. A measurable mapping $\xi: \Omega \rightarrow X$ is said to be an $\mathrm{X}$ valued random variable if the inverse image under the mapping $x$ of every Borel set $B$ of $X$ belongs to $\beta$, that is, $\xi^{-1}(B) \in \beta$ for all $B \in \beta_{X}$. A measurable mapping $\xi: \Omega \rightarrow X$ is said to be a finitelyvalued random variable if it is constant on each finite number of disjoint sets $A_{i} \in \beta$ and is equal to 0 on $\Omega-\left(\cup_{i=1}^{n} A\right)$. $\xi$ is called a simple random variable if it is finitely valued and

$$
\mu\{\omega:\|\xi(\omega)\|>0\}<\infty .
$$

A measurable mapping $\xi: \Omega \rightarrow X$ is said to be a strong random variable if there exists a sequence $\left\{\xi_{n}(\omega)\right\}$ of simple random variables which converges to $\xi(\omega)$ almost surely, that is, there exists a set $A_{0} \in \beta$ with $\mu\left(A_{0}\right)=0$ such that $\lim _{n \rightarrow \infty} \xi_{n}(\omega)=\xi(\omega), \omega \in$
$\Omega-A_{0}$. A measurable mapping $\xi: \Omega \rightarrow X$ is said to be a weak random variable if the function $\xi^{*}(\xi(\omega))$ is a real-valued random variable for each $\xi^{*} \in X^{*}$, the space $X^{*}$ denoting the first normed dual space of $X$. Let $Y$ be another Banach space. A measurable mapping $f: \Omega \times X \rightarrow Y$ is said to be a random mapping if $f(\omega, \xi)=Y(\omega)$ is a Y-valued random variable for every $\xi \in X$. A measurable mapping $f: \Omega \times X \rightarrow Y$ is said to be a continuous random mapping if the set of all $\omega \in \Omega$ for which $f(\omega, \xi)$ is a continuous function of $\xi$ has measure one. A mapping ble $f: \Omega \times X \rightarrow Y$ is said to be demi-continuous at the $\xi \in X$ if $\left\|\xi_{n}-\xi\right\| \rightarrow 0$ implies $f\left(\omega, \xi_{n}\right) \stackrel{\text { weakly }}{\longrightarrow} f(\omega, \xi)$ almost surely. An equation of the type $f(\omega, \xi(\omega))=\xi(\omega)$, where $f: \Omega \times X \rightarrow$ $X$ is a random mapping, is called a random fixed point equation. Any measurable mapping $\xi: \Omega \rightarrow X$ which satisfies the random fixed point equation $f(\omega, \xi(\omega))=\xi(\omega)$ almost surely is said to be a wide sense solution of the fixed point equation. AnyX-valued random variable $\xi(\omega)$ which satisfies $\mu\{\omega: f(\omega, \xi(\omega))=$ $\xi(\omega)\}=1$ is said to be a random solution of the fixed point equation or a random fixed point of $f$. A measurable mapping $\xi: \Omega \rightarrow$ $X$ is called a random fixed point of a random operator $f: \Omega \times X \rightarrow$ $X$ if $\xi(\omega)=f(\omega, \xi(\omega))$ for every $\omega \in \Omega$. A measurable mapping $\xi: \Omega \rightarrow X$ is called a random coincidence of random operators $T, f: \Omega \times X \rightarrow X$ if $T(\omega, \xi(\omega))=f(\omega, \xi(\omega))$ for every $\omega \in \Omega$. A measurable mapping $\xi: \Omega \rightarrow X$ is called a random common fixed point of random operators $T, f: \Omega \times X \rightarrow X$ if $T(\omega, \xi(\omega))=$ $f(\omega, \xi(\omega))=\xi(\omega)$ for every $\omega \in \Omega$.

Example 1 Let $X$ be the set of all real numbers and let $E$ be a nonmeasurable subset of $X$. Let $f: \Omega \times X \rightarrow Y$ be a random mapping defined as $f(\omega, \xi(\omega))=,\xi^{2}(\omega)+\xi(\omega)-1$ for all $\omega \in \Omega$. In this case, the real-valued function $\xi(\omega)$, defined as $\xi(\omega)=1$ for all $\omega \in \Omega$, is a random fixed point of $f$. However, the real-valued function $y(\omega)$ defined as

$$
y(\omega)=\left\{\begin{array}{cc}
-1, & \omega \notin E, \\
1 & \omega \in E
\end{array}\right.
$$

Is a wide sense solution of the fixed point equation $f(\omega, \xi(\omega))=$ $\xi(\omega)$ without being a random fixed point of $f$.

In this paper, we consider the following class of pairs of functions $\mathfrak{F}$ (see [20]). 
Definition 2: A pair of functions $(\varphi, \phi)$ is said to belong to the class $\mathfrak{F}$, if they satisfy the following conditions:

(a1). $\varphi, \phi:[0, \infty) \rightarrow[0, \infty)$;

(a2). For $t, s \in[0, \infty), \varphi(t) \leq \phi(s)$ then $t \leq s$;

(a3). For $\left\{t_{n}\right\}$ and $\left\{s_{n}\right\}$ sequence in $[0, \infty)$ such that $\lim _{n \rightarrow \infty} t_{n}=$ $\lim _{n \rightarrow \infty} s_{n}=a$, if $\varphi\left(t_{n}\right) \leq \phi\left(s_{n}\right)$ for any $n \in \mathbb{N}$, then $a=0$.

Remark 3 (see [20]) Note that, if $(\varphi, \phi) \in \mathfrak{F}$ and $\varphi(t) \leq \phi(t)$, then $t=0$, since we can take $t_{n}=s_{n}=t$ for any $n \in \mathbb{N}$ and by (a3) we deduce that $t=0$.

Example 4 The conditions (a1)-(a3) of the above definition are fulfilled for the functions $\varphi, \phi:[0, \infty) \rightarrow[0, \infty)$ defined by $\varphi(t)=\ln \left(\frac{5 t+1}{12}\right)$ and $\phi(t)=\ln \left(\frac{3 t+1}{12}\right)$ for all $t \in[0, \infty)$.

In the sequel, we present some interesting examples of pairs of functions belonging to the class $\mathfrak{F}$ which will be very important in our study.

Example 5 (see [20]) Let $\varphi:[0, \infty) \rightarrow[0, \infty)$ be a continuous and increasing function such that $\varphi(t)=0$ if and only if $t=0$ (these functions are known in the literature as altering distance functions). Let $\phi:[0, \infty) \rightarrow[0, \infty)$ be a non-decreasing function such that $\phi(t)=0$ if and only if $t=0$ and suppose that $\phi \leq \varphi$. Then the pair $(\varphi, \varphi-\phi) \in \mathfrak{F}$.

An interesting particular case is when $\varphi$ is the identity mapping, $\varphi=1_{[0, \infty)}$ and $\phi:[0, \infty) \rightarrow[0, \infty)$ is a non-decreasing function such that $\phi(t)=0$ if and only if $t=0$ and $\phi(t) \leq t$ for any $t \in[0, \infty)$.

Example 6 (see [20]) Let $S$ be the class of functions defined by $S=\left\{\alpha:[0, \infty) \rightarrow[0,1):\left\{\alpha\left(t_{n}\right) \rightarrow 1 \Rightarrow t_{n} \rightarrow 0\right\}\right\}$. Let us consider the pairs of functions $\left(1_{[0, \infty)}, \alpha 1_{[0, \infty)}\right)$, where $\alpha \in S$ and $\alpha 1_{[0, \infty)}$ is defined by $\left(\alpha 1_{[0, \infty)}\right)(t)=\alpha(t) t$,for $t \in[0, \infty)$. Then $\left(1_{[0, \infty)}, \alpha 1_{[0, \infty)}\right) \in \mathfrak{F}$.

Remark 7 (see [20]) Suppose that $g:[0, \infty) \rightarrow[0, \infty)$ is an increasing function and $(\varphi, \phi) \in \mathfrak{F}$. Then it is easily seen that the pair $(g \circ \varphi, g \circ \phi) \in \mathfrak{F}$.

Definition 8 (see [4]) Let $(X, \preceq)$ is a partially ordered set and $f: X \rightarrow X$ is said to be monotone non-decreasing if for all, $y \in X, x \leq y \Rightarrow f x \leq f y$.

Definition 9 (see [2], [6]) Let $(X, \preceq)$ be a partially ordered set and $T$ and $f$ be two self maps on $X$. An ordered pair $(T, f)$ is said to be weakly increasing if $T x \leq f T x$ and $f x \leq T f x$ for all $x \in X$.

\section{Main results}

In this section, first we introduce the notion of monotone nondecreasing and weakly increasing random operators.

Definition 10 Let $(X, \preceq, d)$ is a partially ordered separable metric space.

1) A random operator $f: \Omega \times X \rightarrow X$ is said to be monotone non-decreasing if for all $x, y \in X$,

$$
x \leq y \Rightarrow f(\omega, x(\omega)) \leq f(\omega, y(\omega)), \omega \in \Omega .
$$

2) Two random operators $T, f: \Omega \times X \rightarrow X$ is said to be weakly increasing if

$$
\begin{aligned}
& T(\omega, x(\omega)) \leq f(\omega, T(\omega, x(\omega))) \\
\text { and } \quad & f(\omega, x(\omega)) \leq T(\omega, f(\omega, x(\omega)))
\end{aligned}
$$

for all $x \in X$ and $\omega \in \Omega$.

Now, we give our main result.

Theorem 11 Let $(X, \preceq)$ is a partially ordered set. Suppose that there exist a metric $d$ on $X$ such that $(X, d)$ be a complete separable metric space and $(\Omega, \Sigma, \mu)$ is a complete probability measure space. Let $T, f: \Omega \times X \rightarrow X$ be two mappings such that

(i) $T(\omega,$.$) and f(\omega,$.$) are continuous for all \omega \in \Omega$;

(ii) $T(., x)$ and $f(., x)$ are measurable mapping for all $x \in X$;

(iii) The pair $(T, f)$ is weakly increasing such that there exists a pair of functions $(\varphi, \phi) \in \mathfrak{F}$ satisfying for all comparable elements $x, y \in X$,

$$
\begin{aligned}
& \varphi(d(T(\omega, x), f(\omega, y))) \\
& \quad \leq \max \left\{\phi(d(x, y)), \phi\left(\frac{d(y, f(\omega, y))[1+d(x, T(\omega, x))]}{1+d(x, y)}\right)\right\}
\end{aligned}
$$

Also suppose either

a) $T$ or $f$ is continuous or

b) $X$ has the following property:

If $\left\{x_{n}\right\}$ is non-decreasing sequence in $X$ such that $x_{n} \rightarrow u$, then $x_{n} \preceq u$, for all $n \in \mathbb{N}$.

Then $T$ and $f$ have a common fixed point.

Proof Let the function $\xi_{0}(\omega): \Omega \rightarrow X$ be an arbitrary measurable mapping. We can define a sequence of measurable mappings $\left\{\xi_{n}(\omega)\right\}$ from $\Omega$ to $X$ as following:

$\xi_{2 n+1}(\omega)=T\left(\omega, \xi_{2 n}(\omega)\right)$,

$\xi_{2 n+2}(\omega)=f\left(\omega, \xi_{2 n+1}(\omega)\right), \omega \in \Omega, n=0,1,2, \ldots$

Since the pair $(T, f)$ is weakly increasing mappings, we have

$$
\begin{aligned}
\xi_{1}(\omega)=T\left(\omega, \xi_{0}(\omega)\right) & \leq f\left(\omega, T\left(\omega, \xi_{0}(\omega)\right)\right) \\
& =f\left(\omega, \xi_{1}(\omega)\right)=\xi_{2}(\omega), \\
\xi_{2}(\omega)=T\left(\omega, \xi_{1}(\omega)\right) & \leq f\left(\omega, T\left(\omega, \xi_{1}(\omega)\right)\right) \\
& =f\left(\omega, \xi_{2}(\omega)\right)=\xi_{3}(\omega),
\end{aligned}
$$

Continuing this process, we get

$$
\begin{aligned}
\xi_{2 n+1}(\omega)=T\left(\omega, \xi_{2 n}(\omega)\right) & \leq f\left(\omega, T\left(\omega, \xi_{2 n}(\omega)\right)\right) \\
& =f\left(\omega, \xi_{2 n+1}(\omega)\right)=\xi_{2 n+2}(\omega) \\
\xi_{2 n+2}(\omega)=T\left(\omega, \xi_{2 n+1}(\omega)\right) & \leq f\left(\omega, T\left(\omega, \xi_{2 n+1}(\omega)\right)\right) \\
& =f\left(\omega, \xi_{2 n+2}(\omega)\right)=\xi_{2 n+3}(\omega)
\end{aligned}
$$

Thus for all $n \geq 1$, we have $\xi_{n}(\omega) \leq \xi_{n+1}(\omega)$. Without loss of the generality, we can assume that $\xi_{n}(\omega) \neq \xi_{n+1}(\omega)$ and since $\xi_{2 n}(\omega)$ and $\xi_{2 n+1}(\omega)$ are comparable, applying the contractive condition (1), we have

$$
\begin{aligned}
\varphi\left(d\left(\xi_{2 n+2}(\omega), \xi_{2 n+1}(\omega)\right)\right) \\
\quad=\varphi\left(d\left(T\left(\omega, \xi_{2 n+1}(\omega)\right), f\left(\omega, \xi_{2 n}(\omega)\right)\right)\right) \\
\leq \max \left\{\phi\left(d\left(\xi_{2 n+1}(\omega), \xi_{2 n}(\omega)\right)\right),\right. \\
\left.\phi\left(\frac{d\left(\xi_{2 n}(\omega), f\left(\omega, \xi_{2 n}(\omega)\right)\right)\left[1+d\left(\xi_{2 n+1}(\omega), T\left(\omega, \xi_{2 n+1}(\omega)\right)\right)\right]}{1+d\left(\xi_{2 n+1}(\omega), \xi_{2 n}(\omega)\right)}\right)\right\} \\
=\max \left\{\phi\left(d\left(\xi_{2 n+1}(\omega), \xi_{2 n}(\omega)\right)\right),\right. \\
\left.\phi\left(\frac{d\left(\xi_{2 n}(\omega), \xi_{2 n+1}(\omega)\right)\left[1+d\left(\xi_{2 n+1}(\omega), \xi_{2 n+2}(\omega)\right)\right]}{1+d\left(\xi_{2 n+1}(\omega), \xi_{2 n}(\omega)\right)}\right)\right\}
\end{aligned}
$$

Now, we can distinguish two cases.

Case I. Consider

$$
\begin{aligned}
\max & \left\{\phi\left(d\left(\xi_{2 n}(\omega), \xi_{2 n+1}(\omega)\right)\right),\right. \\
& \left.\phi\left(\frac{d\left(\xi_{2 n}(\omega), \xi_{2 n+1}(\omega)\right)\left[1+d\left(\xi_{2 n+1}(\omega), \xi_{2 n+2}(\omega)\right)\right]}{1+d\left(\xi_{2 n+1}(\omega), \xi_{2 n}(\omega)\right)}\right)\right\} \\
= & \phi\left(d\left(\xi_{2 n}(\omega), \xi_{2 n+1}(\omega)\right)\right)
\end{aligned}
$$

In this case from (4), we have

$$
\varphi\left(d\left(\xi_{2 n+1}(\omega), \xi_{2 n+2}(\omega)\right)\right) \leq \phi\left(d\left(\xi_{2 n}(\omega), \xi_{2 n+1}(\omega)\right)\right)
$$

Since $(\varphi, \phi) \in \mathfrak{F}$, we deduce that

$$
d\left(\xi_{2 n+1}(\omega), \xi_{2 n+2}(\omega)\right) \leq d\left(\xi_{2 n}(\omega), \xi_{2 n+1}(\omega)\right) .
$$

\section{Case II. If}

$$
\begin{aligned}
\max & \left\{\phi\left(d\left(\xi_{2 n}(\omega), \xi_{2 n+1}(\omega)\right)\right),\right. \\
& \left.\phi\left(\frac{d\left(\xi_{2 n}(\omega), \xi_{2 n+1}(\omega)\right)\left[1+d\left(\xi_{2 n+1}(\omega), \xi_{2 n+2}(\omega)\right)\right]}{1+d\left(\xi_{2 n+1}(\omega), \xi_{2 n}(\omega)\right)}\right)\right\} \\
= & \phi\left(\frac{d\left(\xi_{2 n}(\omega), \xi_{2 n+1}(\omega)\right)\left[1+d\left(\xi_{2 n+1}(\omega), \xi_{2 n+2}(\omega)\right)\right]}{1+d\left(\xi_{2 n+1}(\omega), \xi_{2 n}(\omega)\right)}\right)
\end{aligned}
$$

In this case from (4), we have

$$
\begin{aligned}
\varphi( & \left.d\left(\xi_{2 n+1}(\omega), \xi_{2 n+2}(\omega)\right)\right) \\
& \leq \phi\left(\frac{d\left(\xi_{2 n}(\omega), \xi_{2 n+1}(\omega)\right)\left[1+d\left(\xi_{2 n+1}(\omega), \xi_{2 n+2}(\omega)\right)\right]}{1+d\left(\xi_{2 n+1}(\omega), \xi_{2 n}(\omega)\right)}\right)
\end{aligned}
$$

Since $(\varphi, \phi) \in \mathfrak{F}$, we get

$$
d\left(\xi_{2 n+1}(\omega), \xi_{2 n+2}(\omega)\right) \leq \frac{d\left(\xi_{2 n}(\omega), \xi_{2 n+1}(\omega)\right)\left[1+d\left(\xi_{2 n+1}(\omega), \xi_{2 n+2}(\omega)\right)\right]}{1+d\left(\xi_{2 n+1}(\omega), \xi_{2 n}(\omega)\right)}
$$

Since $d\left(\xi_{2 n+1}(\omega), \xi_{2 n+2}(\omega)\right) \neq 0$, from the last inequality, we have

$$
d\left(\xi_{2 n+1}(\omega), \xi_{2 n+2}(\omega)\right) \leq d\left(\xi_{2 n}(\omega), \xi_{2 n+1}(\omega)\right)
$$


In both cases, we conclude that the sequence $\left\{d\left(\xi_{2 n}(\omega), \xi_{2 n+1}(\omega)\right)\right\}$ is a decreasing sequence of non-negative real numbers and is bounded below, there exists $r(\omega) \geq 0$ such that

$$
\lim _{n \rightarrow \infty} d\left(\xi_{2 n}(\omega), \xi_{2 n+1}(\omega)\right)=r(\omega), \omega \in \Omega
$$

Now, we shall show that $r(\omega)=0$.

Denote

$$
\begin{aligned}
& A=\{n \in \mathbb{N}: n \text { satisfies (5) }\}, \\
& B=\{n \in \mathbb{N}: n \text { satisfies (7) }\} .
\end{aligned}
$$

By (4), we have $\operatorname{Card} A=\infty$ or $\operatorname{CardB}=\infty$. Let us suppose that $\operatorname{CardC}=\infty$. Then there exists infinitely natural numbers $n$ satisfying inequality (6). Since $(\varphi, \phi) \in \mathfrak{F}$, we infer from (9) and condition (a3) that $r(\omega)=0, \omega \in \Omega$. On the other hand, if $\operatorname{CardB}=\infty$, then from (4), we can find infinitely many $n \in \mathbb{N}$ satisfying inequality (8). Since $(\phi, \varphi) \in \mathfrak{F}$, we obtain

$d\left(\xi_{2 n+1}(\omega), \xi_{2 n+2}(\omega)\right) \leq \frac{d\left(\xi_{2 n}(\omega), \xi_{2 n+1}(\omega)\right)\left[1+d\left(\xi_{2 n+1}(\omega), \xi_{2 n+2}(\omega)\right)\right]}{1+d\left(\xi_{2 n+1}(\omega), \xi_{2 n}(\omega)\right)}$ For infinitely many $n \in \mathbb{N}$. Letting the limit as $n \rightarrow \infty$ and taking into account that (9), we deduce that $r(\omega) \leq r(\omega) \frac{1+r(\omega)}{1+r(\omega)}$ and consequently, we obtain $r(\omega)=0, \omega \in \Omega$.Therefore, in both cases we have

$$
\lim _{n \rightarrow \infty} d\left(\xi_{2 n}(\omega), \xi_{2 n+1}(\omega)\right)=0, \omega \in \Omega .
$$

Now, we will show that for $\omega \in \Omega,\left\{\xi_{n}(\omega)\right\}$ is a Cauchy sequence, it is sufficient to prove that $\left\{\xi_{2 n}(\omega)\right\}$ is a Cauchy sequence. We proceed by negation, suppose that $\left\{\xi_{2 n}(\omega)\right\}$ is not a Cauchy sequence, then there exist $\epsilon(\omega)>0$ for which we can find two subsequences of positive integers $\left\{m_{i}\right\}$ and $\left\{n_{i}\right\}$ for positive integer $i$, we

$$
m_{i}>n_{i}>i, d\left(\xi_{2 n_{i}}(\omega), \xi_{2 m_{i}}(\omega)\right) \geq \epsilon(\omega), i \geq 1, \omega \in \Omega
$$

Further, we can choose $m_{i}$ to be smallest integer with $m_{i}>n_{i}$ for which (11) holds. Then

$$
d\left(\xi_{2 n_{i}}(\omega), \xi_{2 m_{i}-2}(\omega)\right)<\epsilon(\omega)
$$

Using (10), (11) and the triangle inequality, we obtain

$$
\begin{aligned}
\epsilon(\omega) & \leq d\left(\xi_{2 n_{i}}(\omega), \xi_{2 m_{i}}(\omega)\right) \\
& \leq d\left(\xi_{2 n_{i}}(\omega), \xi_{2 m_{i}-2}(\omega)\right)+d\left(\xi_{2 m_{i}-2}(\omega), \xi_{2 m_{i}-1}(\omega)\right) \\
& +d\left(\xi_{2 m_{i}-1}(\omega), \xi_{2 m_{i}}(\omega)\right) \\
& \leq \epsilon(\omega)+d\left(\xi_{2 m_{i}-2}(\omega), \xi_{2 m_{i}-1}(\omega)\right) \\
& +d\left(\xi_{2 m_{i}-1}(\omega), \xi_{2 m_{i}}(\omega)\right)
\end{aligned}
$$

On letting the limit as $i \rightarrow \infty$ in the above inequality and using (9), we get

$$
\lim _{i \rightarrow \infty} d\left(\xi_{2 n_{i}}(\omega), \xi_{2 m_{i}}(\omega)\right)=\epsilon(\omega), \omega \in \Omega
$$

In addition, by the triangle inequality, we have

$$
\begin{aligned}
d\left(\xi_{2 n_{i}}(\omega), \xi_{2 m_{i}}(\omega)\right) & \leq d\left(\xi_{2 n_{i}}(\omega), \xi_{2 n_{i}-1}(\omega)\right) \\
& +d\left(\xi_{2 n_{i}-1}(\omega), \xi_{2 m_{i}-1}(\omega)\right) \\
& +d\left(\xi_{2 m_{i}-1}(\omega), \xi_{2 m_{i}}(\omega)\right)
\end{aligned}
$$

And

$$
\begin{aligned}
d\left(\xi_{2 n_{i}-1}(\omega), \xi_{2 m_{i}-1}(\omega)\right) & \leq d\left(\xi_{2 n_{i}-1}(\omega), \xi_{2 n_{i}}(\omega)\right) \\
& +d\left(\xi_{2 n_{i}}(\omega), \xi_{2 m_{i}}(\omega)\right) \\
& +d\left(\xi_{2 m_{i}}(\omega), \xi_{2 m_{i}-1}(\omega)\right)
\end{aligned}
$$

Letting the limit as $i \rightarrow \infty$ in the above two inequality, using (9) and (14), we get

$$
\lim _{i \rightarrow \infty} d\left(\xi_{2 n_{i}-1}(\omega), \xi_{2 m_{i}-1}(\omega)\right)=\epsilon(\omega), \omega \in \Omega
$$

Since $m_{i}>n_{i}$ and $\xi_{2 n_{i-1}}(\omega)$ and $\xi_{2 m_{i}-1}(\omega)$ are comparable, then by contractive condition (1), we get

$$
\begin{aligned}
\varphi( & \left.d\left(\xi_{2 n_{i}}(\omega), \xi_{2 m_{i}}(\omega)\right)\right) \\
& =\varphi\left(d\left(T\left(\omega, \xi_{2 n_{i}-1}(\omega)\right), f\left(\omega, \xi_{2 m_{i}-1}(\omega)\right)\right)\right) \\
& \leq \max \left\{\phi\left(d\left(\xi_{2 n_{i}-1}(\omega), \xi_{2 m_{i}-1}(\omega)\right)\right),\right.
\end{aligned}
$$

$$
\begin{aligned}
& \left.\phi\left(\frac{d\left(\xi_{2 m_{i^{-1}}}(\omega), f\left(\omega, \xi_{2 m_{i^{-1}}}(\omega)\right)\right)\left[1+d\left(\xi_{2 n_{i^{-1}}}(\omega), T\left(\omega, \xi_{2 n_{i^{-1}}}(\omega)\right)\right)\right]}{1+d\left(\xi_{2 n_{i^{-1}}}(\omega), \xi_{2 m_{i^{-1}}}(\omega)\right)}\right)\right\} \\
& =\max \left\{\phi\left(d\left(\xi_{2 n_{i}-1}(\omega), \xi_{2 m_{i^{-1}}}(\omega)\right)\right)\right. \\
& \left.\phi\left(\frac{d\left(\xi_{2 m_{i^{-1}}}(\omega), \xi_{2 m_{i}}(\omega)\right)\left[1+d\left(\xi_{2 n_{i^{-1}}}(\omega), \xi_{2 n_{i}}(\omega)\right)\right]}{1+d\left(\xi_{2 n_{i^{-1}}}(\omega), \xi_{2 m_{i^{-1}}}(\omega)\right)}\right)\right\}
\end{aligned}
$$

Let us put

$$
\begin{array}{r}
C=\left\{i \in \mathbb{N}: \varphi\left(d\left(\xi_{2 n_{i}}(\omega), \xi_{2 m_{i}}(\omega)\right)\right)\right. \\
\left.\leq \phi\left(d\left(\xi_{2 n_{i}-1}(\omega), \xi_{2 m_{i}-1}(\omega)\right)\right)\right\}, \\
D=\left\{i \in \mathbb{N}: \varphi\left(d\left(\xi_{2 n_{i}}(\omega), \xi_{2 m_{i}}(\omega)\right)\right)\right. \\
\left.\leq \phi\left(\frac{d\left(\xi_{2 m_{i^{-1}}}(\omega), \xi_{2 m_{i}}(\omega)\right)\left[1+d\left(\xi_{2 n_{i}-1}(\omega), \xi_{2 n_{i}}(\omega)\right)\right]}{1+d\left(\xi_{2 n_{i}-1}(\omega), \xi_{2 m_{i}-1}(\omega)\right)}\right)\right\}
\end{array}
$$

From (16), we have $\operatorname{CardC}=\infty$ or $\operatorname{CardD}=\infty$. Let us supposethat $\operatorname{CardC}=\infty$. Then there exists infinitely many $i \in \mathbb{N}$ satisfying

$$
\varphi\left(d\left(\xi_{2 n_{i}}(\omega), \xi_{2 m_{i}}(\omega)\right)\right) \leq \phi\left(d\left(\xi_{2 n_{i}-1}(\omega), \xi_{2 m_{i}-1}(\omega)\right)\right)
$$

Since $(\varphi, \phi) \in \mathfrak{F}$, we infer from (14), (15) and condition (a3) that $\epsilon(\omega)=0$. This is a contradiction.

On the other hand, if $\operatorname{CardD}=\infty$, then we can find infinitely many $i \in \mathbb{N}$ satisfying

$$
\begin{aligned}
& \varphi\left(d\left(\xi_{2 n_{i}}(\omega), \xi_{2 m_{i}}(\omega)\right)\right) \\
& \leq \phi\left(\frac{d\left(\xi_{2 m_{i^{-1}}}(\omega), \xi_{2 m_{i}}(\omega)\right)\left[1+d\left(\xi_{2 n_{i^{-1}}}(\omega), \xi_{2 n_{i}}(\omega)\right)\right]}{1+d\left(\xi_{2 n_{i^{-1}}}(\omega), \xi_{2 m_{i^{-1}}}(\omega)\right)}\right)
\end{aligned}
$$

and since $(\varphi, \phi) \in \mathfrak{F}$, we obtain

$$
\begin{aligned}
d\left(\xi_{2 n_{i}}(\omega),\right. & \left.\xi_{2 m_{i}}(\omega)\right) \\
& \leq \frac{d\left(\xi_{2 m_{i}-1}(\omega), \xi_{2 m_{i}}(\omega)\right)\left[1+d\left(\xi_{2 n_{i^{-1}}}(\omega), \xi_{2 n_{i}}(\omega)\right)\right]}{1+d\left(\xi_{2 n_{i^{-1}}}(\omega), \xi_{2 m_{i^{-1}}}(\omega)\right)}
\end{aligned}
$$

Taking the limit as $i \rightarrow \infty$ in above inequality, using (9), (14) and (15), we obtain $\epsilon(\omega) \leq 0$, which is a contradiction. Therefore, since in both possibilities $\operatorname{CardC}=\infty$, and $\operatorname{CardD}=\infty$, we obtain a contradiction, we deduce that $\left\{\xi_{2 n}(\omega)\right\}$ is a Cauchy sequence in $X$ and so is $\left\{\xi_{n}(\omega)\right\}$, then there exists $\xi(\omega): \Omega \rightarrow X$ such that

Now, if $T$ is continuous, then

$$
\lim _{n \rightarrow \infty} \xi_{n}(\omega)=\xi(\omega) .
$$

$$
\begin{aligned}
\xi(\omega) & =\lim _{n \rightarrow \infty} \xi_{2 n+1}(\omega) \\
& =\lim _{n \rightarrow \infty} T\left(\omega, \xi_{2 n}(\omega)\right)=T(\omega, \xi(\omega)) .
\end{aligned}
$$

Also since $\xi(\omega) \preceq \xi(\omega)$, applying contractive condition (1) and using (18), we have

$$
\begin{aligned}
\varphi(d(\xi(\omega), f(\omega, \xi(\omega)))) \\
=\varphi(d(T(\omega, \xi(\omega)), f(\omega, \xi(\omega)))) \\
\leq \max \{\phi(d(\xi(\omega), \xi(\omega))), \\
\left.\phi\left(\frac{d(\xi(\omega), f(\omega, \xi(\omega)))[1+d(\xi(\omega), T(\omega, \xi(\omega)))]}{1+d(\xi(\omega), \xi(\omega))}\right)\right\} \\
=\max \{\phi(0), \phi(d(\xi(\omega), f(\omega, \xi(\omega))))\}
\end{aligned}
$$

Consider

$$
\max \{\phi(0), \phi(d(\xi(\omega), f(\omega, \xi(\omega))))\}=\phi(0)
$$

Then from (19), we have

$$
\varphi(d(\xi(\omega), f(\omega, \xi(\omega)))) \leq \phi(0)
$$

Since $(\varphi, \phi) \in \mathfrak{F}$, we infer that $d(\xi(\omega), f(\omega, \xi(\omega)))=0$ and so $\xi(\omega)=f(\omega, \xi(\omega))$.

Consider

$$
\begin{aligned}
\max \{\phi(0), \phi(d(\xi(\omega), f(\omega, \xi(\omega)))))\} \\
=\phi(d(\xi(\omega), f(\omega, \xi(\omega))))
\end{aligned}
$$


Then from (19), we have

$$
\varphi(d(\xi(\omega), f(\omega, \xi(\omega)))) \leq \phi(d(\xi(\omega), f(\omega, \xi(\omega))))
$$

Since $(\varphi, \phi) \in \mathfrak{F}$, by Remark 3, we deduce that

$$
d(\xi(\omega), f(\omega, \xi(\omega)))=0
$$

and so $\xi(\omega)=f(\omega, \xi(\omega))$. In both cases, we obtain

$$
\xi(\omega)=f(\omega, \xi(\omega))
$$

From (18) and (20), we have

$$
\xi(\omega)=f(\omega, \xi(\omega))=T(\omega, \xi(\omega))
$$

Similarly, we obtain the same result if $f$ is continuous.

Now, if the condition (b) is satisfied. Since $\lim _{n \rightarrow \infty} \xi_{2 n-1}(\omega)=$ $\xi(\omega)$, then we have $\xi_{2 n-1}(\omega) \leq \xi(\omega)$. Thus, by (1), we have

$$
\begin{aligned}
\varphi\left(d\left(\xi_{2 n}, f(\omega, \xi(\omega))\right)\right) \\
=\varphi\left(d\left(T\left(\omega, \xi_{2 n-1}\right), f(\omega, \xi(\omega))\right)\right) \\
\leq \max \left\{\phi\left(d\left(\xi_{2 n-1}, \xi(\omega)\right)\right),\right. \\
\left.\phi\left(\frac{d(\xi(\omega), f(\omega, \xi(\omega)))\left[1+d\left(\xi_{2 n-1}, T\left(\omega, \xi_{2 n-1}\right)\right)\right]}{1+d\left(\xi_{2 n-1}, \xi(\omega)\right)}\right)\right\}
\end{aligned}
$$

Put

$$
\begin{aligned}
& E=\{n \in \mathbb{N}: \varphi(\left.\left.d\left(\xi_{2 n}, f(\omega, \xi(\omega))\right)\right) \leq \phi\left(d\left(\xi_{2 n-1}, \xi(\omega)\right)\right)\right\}, \\
& F=\left\{n \in \mathbb{N}: \varphi\left(d\left(\xi_{2 n}, f(\omega, \xi(\omega))\right)\right)\right. \\
&\left.\leq \phi\left(\frac{d(\xi(\omega), f(\omega, \xi(\omega)))\left[1+d\left(\xi_{2 n-1}, T\left(\omega, \xi_{2 n-1}\right)\right)\right]}{1+d\left(\xi_{2 n-1}, \xi(\omega)\right)}\right)\right\}
\end{aligned}
$$

From (21), we have $\operatorname{CardE}=\infty$ or $\operatorname{CardF}=\infty$. Let us supposethat $\operatorname{CardE}=\infty$. Then there exists infinitely many $n \in \mathbb{N}$ satisfying

$$
\varphi\left(d\left(\xi_{2 n}, f(\omega, \xi(\omega))\right)\right) \leq \phi\left(d\left(\xi_{2 n-1}, \xi(\omega)\right)\right)
$$

Since $(\varphi, \phi) \in \mathfrak{F}$, we have

$$
d\left(\xi_{2 n}, f(\omega, \xi(\omega))\right) \leq d\left(\xi_{2 n-1}, \xi(\omega)\right)
$$

Letting the limit as $n \rightarrow \infty$ in above inequality and using (17), we have $d(\xi(\omega), f(\omega, \xi(\omega)))=0$ and consequently, $f(\omega, \xi(\omega))=$ $\xi(\omega)$.

On the other hand, if $\operatorname{CardF}=\infty$, then we can find infinitely many $i \in \mathbb{N}$ satisfying

$$
\begin{aligned}
\varphi\left(d\left(\xi_{2 n}, f(\omega, \xi(\omega))\right)\right) \\
\quad \leq \phi\left(\frac{d(\xi(\omega), f(\omega, \xi(\omega)))\left[1+d\left(\xi_{2 n-1}, T\left(\omega, \xi_{2 n-1}\right)\right)\right]}{1+d\left(\xi_{2 n-1}, \xi(\omega)\right)}\right)
\end{aligned}
$$

Where, to simplify our considerations, we will denote the subsequence by the same symbol $T\left(\omega, \xi_{2 n-1}\right)$. By (2), (10) and (17), we have

$$
\begin{aligned}
& \lim _{n \rightarrow \infty}\left(\frac{d(\xi(\omega), f(\omega, \xi(\omega)))\left[1+d\left(\xi_{2 n-1}, T\left(\omega, \xi_{2 n-1}\right)\right)\right]}{1+d\left(\xi_{2 n-1}, \xi(\omega)\right)}\right) \\
& =\lim _{n \rightarrow \infty}\left(\frac{d(\xi(\omega), f(\omega, \xi(\omega)))\left[1+d\left(\xi_{2 n-1}, \xi_{2 n}\right)\right]}{1+d\left(\xi_{2 n-1}, \xi(\omega)\right)}\right) \\
& =d(\xi(\omega), f(\omega, \xi(\omega))) \\
& =\lim _{n \rightarrow \infty} d\left(\xi_{2 n}, f(\omega, \xi(\omega))\right)
\end{aligned}
$$

Since $(\phi, \varphi) \in \mathfrak{F}$, we infer from (22) and condition (a3) that $d(\xi(\omega), f(\omega, \xi(\omega)))=0$ and consequently, $\xi(\omega)=f(\omega, \xi(\omega))$. From the abovecase, we deduce that $\xi(\omega)=f(\omega, \xi(\omega))$.

Similarly, we can show that $\xi(\omega)=T(\omega, \xi(\omega))$. The proof of the theorem is completed.

By Theorem 11, we obtain the following corollaries.

Corollary 12 Let $(X, \preceq)$ is a partially ordered set. Suppose that there exist a metric $d$ on $X$ such that $(X, d)$ be a complete separable metric space and $(\Omega, \Sigma, \mu)$ is a complete probability measure space. Let $T, f: \Omega \times X \rightarrow X$ be two mappings such that

(i) $T(\omega,$.$) and f(\omega,$.$) are continuous for all \omega \in \Omega$;

(ii) $T(., x)$ and $f(., x)$ are measurable mapping for all $x \in X$; (iii) The pair $(T, f)$ is weakly increasing such that for all comparable elements $x, y \in X$, satisfying

$$
d(T(\omega, x), f(\omega, y)) \leq \alpha \frac{d(y, f(\omega, y))[1+d(x, T(\omega, x))]}{1+d(x, y)}+\beta d(x, y)
$$

where $\alpha, \beta>0$ and $\alpha+\beta<1$.

Also suppose either

a) $T$ or $f$ is continuous or

b) $X$ has the following property:

If $\left\{x_{n}\right\}$ is non-decreasing sequence in $X$ such that $x_{n} \rightarrow u$, then $x_{n} \preceq u$, for all $n \in \mathbb{N}$.

Then $T$ and $f$ have a common fixed point.

Proof: Since

$$
\begin{aligned}
& d(T(\omega, x), f(\omega, y)) \leq \alpha \frac{d(y, f(\omega, y))[1+d(x, T(\omega, x))]}{1+d(x, y)}+\beta d(x, y) \\
& \quad \leq(\alpha+\beta) \max \left\{d(x, y), \frac{d(y, f(\omega, y))[1+d(x, T(\omega, x))]}{1+d(x, y)}\right\} \\
& \quad=\max \left\{(\alpha+\beta) d(x, y),(\alpha+\beta) \frac{d(y, f(\omega, y))[1+d(x, T(\omega, x))]}{1+d(x, y)}\right\}
\end{aligned}
$$

for all comparable elements $x, y \in X$, where $\alpha+\beta<1$. This condition is a particular case of the contractive condition appearing in Theorem 11 with the pair of functions $(\varphi, \phi) \in \mathfrak{F}$, given by $\varphi=1_{[0, \infty)}$ and $\phi=(\alpha+\beta) 1_{[0, \infty)}$ (see Example 6).

Corollary 13 Let $(X, \preceq)$ is a partially ordered set. Suppose that there exist a metric $d$ on $X$ such that $(X, d)$ be a complete separable metric space and $(\Omega, \Sigma, \mu)$ is a complete probability measure space. Let $T, f: \Omega \times X \rightarrow X$ be two mappings such that

(i) $T(\omega,$.$) and f(\omega,$.$) are continuous for all \omega \in \Omega$;

(ii) $T(., x)$ and $f(., x)$ are measurable mapping for all $x \in X$;

(iii) The pair $(T, f)$ is weakly increasing such that there exists a pair of functions $(\varphi, \phi) \in \mathfrak{F}$ satisfying for all comparable elements $x, y \in X$,

$$
\varphi(d(T(\omega, x), f(\omega, y))) \leq \phi(d(x, y))
$$

Also suppose either

a) $T$ or $f$ is continuous or

b) $X$ has the following property:

If $\left\{x_{n}\right\}$ is non-decreasing sequence in $X$ such that $x_{n} \rightarrow u$, then $x_{n} \leq u$, for all $n \in \mathbb{N}$.

Then $T$ and $f$ have a common fixed point.

Corollary 14 Let $(X, \preceq)$ is a partially ordered set. Suppose that there exist a metric $d$ on $X$ such that $(X, d)$ be a complete separable metric space and $(\Omega, \Sigma, \mu)$ is a complete probability measure space. Let $T, f: \Omega \times X \rightarrow X$ be two mappings such that

(i) $T(\omega,$.$) and f(\omega,$.$) are continuous for all \omega \in \Omega$;

(ii) $T(., x)$ and $f(., x)$ are measurable mapping for all $x \in X$;

(iii) The pair $(T, f)$ is weakly increasing such that there exists a pair of functions $(\varphi, \phi) \in \mathfrak{F}$ satisfying for all comparable elements $x, y \in X$,

$$
\varphi(d(T(\omega, x), f(\omega, y))) \leq \phi\left(\frac{d(y, f(\omega, y))[1+d(x, T(\omega, x))]}{1+d(x, y)}\right)
$$

Also suppose either

a) $T$ or $f$ is continuous or

b) $X$ has the following property:

If $\left\{x_{n}\right\}$ is non-decreasing sequence in $X$ such that $x_{n} \rightarrow u$, then $x_{n} \leq u$, for all $n \in \mathbb{N}$.

Then $T$ and $f$ have a common fixed point.

Taking into account Example 5, we have the following corollary.

Corollary 15 Let $(X, \preceq)$ is a partially ordered set. Suppose that there exist a metric $d$ on $X$ such that $(X, d)$ be a complete separable metric space and $(\Omega, \Sigma, \mu)$ is a complete probability measure space. Let $T, f: \Omega \times X \rightarrow X$ be two mappings such that

(i) $T(\omega,$.$) and f(\omega,$.$) are continuous for all \omega \in \Omega$;

(ii) $T(., x)$ and $f(., x)$ are measurable mapping for all $x \in X$;

(iii) The pair $(T, f)$ is weakly increasing such that there exists a pair of functions $(\varphi, \phi) \in \mathfrak{F}$ satisfying for all comparable elements $x, y \in X$, 


$$
\begin{gathered}
\varphi(d(T(\omega, x), f(\omega, y))) \leq \max \{\varphi(d(x, y))-\phi(d(x, y)), \\
\left.\varphi\left(\frac{d(y, f(\omega, y))[1+d(x, T(\omega, x))]}{1+d(x, y)}\right)-\phi\left(\frac{d(y, f(\omega, y))[1+d(x, T(\omega, x))]}{1+d(x, y)}\right)\right\}
\end{gathered}
$$

Also suppose either

a) $T$ or $f$ is continuous or

b) $X$ has the following property:

If $\left\{x_{n}\right\}$ is non-decreasing sequence in $X$ such that $x_{n} \rightarrow u$, then $x_{n} \preceq u$, for all $n \in \mathbb{N}$.

Then $T$ and $f$ have a common fixed point.

Corollary 15 has the following consequences.

Corollary 16 Let $(X, \preceq)$ is a partially ordered set. Suppose that there exist a metric $d$ on $X$ such that $(X, d)$ be a complete separable metric space and $(\Omega, \Sigma, \mu)$ is a complete probability measure space. Let $T, f: \Omega \times X \rightarrow X$ be two mappings such that

(i) $T(\omega,$.$) and f(\omega,$.$) are continuous for all \omega \in \Omega$;

(ii) $T(., x)$ and $f(., x)$ are measurable mapping for all $x \in X$;

(iii) The pair $(T, f)$ is weakly increasing such that there exists a pair of functions $(\varphi, \phi) \in \mathfrak{F}$ satisfying for all comparable elements $x, y \in X$,

$$
\varphi(d(T(\omega, x), f(\omega, y))) \leq \varphi(d(x, y))-\phi(d(x, y))
$$

Also suppose either

a) $\quad T$ or $f$ is continuous or

b) $X$ has the following property:

If $\left\{x_{n}\right\}$ is non-decreasing sequence in $X$ such that $x_{n} \rightarrow u$, then $x_{n} \leq u$, for all $n \in \mathbb{N}$.

Then $T$ and $f$ have a common fixed point.

Corollary 17 Let $(X, \preceq)$ is a partially ordered set. Suppose that there exist a metric $d$ on $X$ such that $(X, d)$ be a complete separable metric space and $(\Omega, \Sigma, \mu)$ is a complete probability measure space. Let $T, f: \Omega \times X \rightarrow X$ be two mappings such that

(i) $T(\omega,$.$) and f(\omega,$.$) are continuous for all \omega \in \Omega$;

(ii) $T(., x)$ and $f(., x)$ are measurable mapping for all $x \in X$;

(iii) The pair $(T, f)$ is weakly increasing such that there exists a pair of functions $(\varphi, \phi) \in \mathfrak{F}$ satisfying for all comparable elements $x, y \in X$,

$$
\begin{aligned}
\varphi(d(T(\omega, x), f(\omega, y))) & \leq \varphi\left(\frac{d(y, f(\omega, y))[1+d(x, T(\omega, x))]}{1+d(x, y)}\right) \\
& -\phi\left(\frac{d(y, f(\omega, y))[1+d(x, T(\omega, x))]}{1+d(x, y)}\right)
\end{aligned}
$$

Also suppose either

a) $\quad T$ or $f$ is continuous or

b) $X$ has the following property:

If $\left\{x_{n}\right\}$ is non-decreasing sequence in $X$ such that $x_{n} \rightarrow u$, then $x_{n} \leq u$, for all $n \in \mathbb{N}$.

Then $T$ and $f$ have a common fixed point.

Taking into account Example 6, we have the following corollary.

Corollary 18 Let $(X, \preceq)$ is a partially ordered set. Suppose that there exist a metric $d$ on $X$ such that $(X, d)$ be a complete separable metric space and $(\Omega, \Sigma, \mu)$ is a complete probability measure space. Let $T, f: \Omega \times X \rightarrow X$ be two mappings such that

(i) $T(\omega,$.$) and f(\omega,$.$) are continuous for all \omega \in \Omega$;

(ii) $T(., x)$ and $f(., x)$ are measurable mapping for all $x \in X$;

(iii) The pair $(T, f)$ is weakly increasing such that such that there exists $\alpha \in S$ (see Example 6) satisfying for all comparable elements $x, y \in X$,

$d(T(\omega, x), f(\omega, y)) \leq \max \{\alpha(d(x, y)) d(x, y)$

$\alpha\left(\frac{d(y, f(\omega, y))[1+d(x, T(\omega, x))]}{1+d(x, y)}\right) \frac{d(y, f(\omega, y))[1+d(x, T(\omega, x))]}{1+d(x, y)}$

Also suppose either

a) $T$ or $f$ is continuous or

b) $X$ has the following property:

If $\left\{x_{n}\right\}$ is non-decreasing sequence in $X$ such that $x_{n} \rightarrow u$, then $x_{n} \leq u$, for all $n \in \mathbb{N}$.

Then $T$ and $f$ have a common fixed point.

A consequence of Corollary 18 is the following corollary.
Corollary 19 Let $(X, \preceq)$ is a partially ordered set. Suppose that there exist a metric $d$ on $X$ such that $(X, d)$ be a complete separable metric space and $(\Omega, \Sigma, \mu)$ is a complete probability measure space. Let $T, f: \Omega \times X \rightarrow X$ be two mappings such that

(i) $T(\omega,$.$) and f(\omega,$.$) are continuous for all \omega \in \Omega$;

(ii) $T(., x)$ and $f(., x)$ are measurable mapping for all $x \in X$;

(iii) The pair $(T, f)$ is weakly increasing such that such that there exists $\alpha \in S$ (see Example 6) satisfying for all comparable elements $x, y \in X$,

$$
d(T(\omega, x), f(\omega, y)) \leq \alpha(d(x, y)) d(x, y)
$$

Also suppose either

a) $T$ or $f$ is continuous or

b) $X$ has the following property:

If $\left\{x_{n}\right\}$ is non-decreasing sequence in $X$ such that $x_{n} \rightarrow u$, then $x_{n} \leq u$, for all $n \in \mathbb{N}$.

Then $T$ and $f$ have a common fixed point.

Corollary 20 Let $(X, \preceq)$ is a partially ordered set. Suppose that there exist a metric $d$ on $X$ such that $(X, d)$ be a complete separable metric space and $(\Omega, \Sigma, \mu)$ is a complete probability measure space. Let $T, f: \Omega \times X \rightarrow X$ be two mappings such that

(i) $T(\omega,$.$) and f(\omega,$.$) are continuous for all \omega \in \Omega$;

(ii) $T(., x)$ and $f(., x)$ are measurable mapping for all $x \in X$;

(iii) The pair $(T, f)$ is weakly increasing such that such that there exists $\alpha \in S$ (see Example 6) satisfying for all comparable elements $x, y \in X$,

$$
\begin{aligned}
& d(T(\omega, x), f(\omega, y)) \\
& \quad \leq \alpha\left(\frac{d(y, f(\omega, y))[1+d(x, T(\omega, x))]}{1+d(x, y)}\right) \frac{d(y, f(\omega, y))[1+d(x, T(\omega, x))]}{1+d(x, y)}
\end{aligned}
$$

Also suppose either

a) $T$ or $f$ is continuous or

b) $X$ has the following property:

If $\left\{x_{n}\right\}$ is non-decreasing sequence in $X$ such that $x_{n} \rightarrow u$, then $x_{n} \preceq u$, for all $n \in \mathbb{N}$.

Then $T$ and $f$ have a common fixed point.

Taking $T=f$ in Theorem 11, we obtain the following Corollary:

Corollary 21 Let $(X, \preceq)$ is a partially ordered set. Suppose that there exist a metric $d$ on $X$ such that $(X, d)$ be a complete separable metric space and $(\Omega, \Sigma, \mu)$ is a complete probability measure space. Let $f: \Omega \times X \rightarrow X$ be two mappings such that

(i) $f(\omega,$.$) is continuous for all \omega \in \Omega$;

(ii) $f(., x)$ is measurable mapping for all $x \in X$;

(iii) The pair $T$ is non-decreasing mapping such that $\xi_{0} \leq$ $f\left(\omega, \xi_{0}(\omega)\right)$ and there exists a pair of functions $(\varphi, \phi) \in$ $\mathfrak{F}$ satisfying for all comparable elements $x, y \in X$,

$$
\begin{aligned}
\varphi(d( & (\omega, x), f(\omega, y))) \\
& \leq \max \left\{\phi(d(x, y)), \phi\left(\frac{d(y, f(\omega, y))[1+d(x, f(\omega, x))]}{1+d(x, y)}\right)\right\}
\end{aligned}
$$

Also suppose either

a) $f$ is continuous or

b) $X$ has the following property:

If $\left\{x_{n}\right\}$ is non-decreasing sequence in $X$ such that $x_{n} \rightarrow u$, then $x_{n} \leq u$, for all $n \in \mathbb{N}$.

Then $T$ has a fixed point.

In what follows, we prove a sufficient condition for the uniqueness of the fixed point in Corollary 21.

Theorem 22 Suppose that:

a) Hypothesis of Corollary 21 hold;

b) For each measurable mappings $\eta(\omega), \zeta(\omega): \Omega \rightarrow X$, there exists a measurable mapping $\xi(\omega): \Omega \rightarrow X$ which is comparable with both $\eta(\omega)$ and $\zeta(\omega)$.

Then $f$ has a unique fixed point.

Proof: By Corollary 21, $f$ has a fixed point. Now we prove that the uniqueness of the fixed point of $f$. Let $\eta(\omega)$ and $\zeta(\omega)$ be two fixed points of $f$.

We consider the following two cases: 
Case. $1 \eta(\omega)$ is comparable to $\zeta(\omega)$. Then $f^{n}(\omega, \eta(\omega))$ is comparable to $f^{n}(\omega, \zeta(\omega))$ for all $n \in \mathbb{N}$. Applying (1), we have

$$
\begin{aligned}
\varphi & (d(\eta(\omega), \zeta(\omega))) \\
& =\varphi\left(d\left(f^{n}(\omega, \eta(\omega)), f^{n}(\omega, \zeta(\omega))\right)\right) \\
& \leq \max \left\{\phi\left(d\left(f^{n-1}(\omega, \eta(\omega)), f^{n-1}(\omega, \zeta(\omega))\right)\right),\right. \\
& \left.\phi\left(\frac{d\left(f^{n-1}(\omega, \zeta(\omega)), f^{n}(\omega, \zeta(\omega))\right)\left[1+d\left(f^{n-1}(\omega, \eta(\omega)), f^{n}(\omega, \eta(\omega))\right)\right]}{1+d\left(f^{n-1}(\omega, \eta(\omega)), f^{n-1}(\omega, \zeta(\omega))\right)}\right)\right\} \\
& =\max \left\{\phi(d(\eta(\omega), \zeta(\omega))), \phi\left(\frac{d(\zeta(\omega), \zeta(\omega))[1+d(\eta(\omega), \eta(\omega))]}{1+d(\eta(\omega), \zeta(\omega))}\right)\right\} \\
& =\max \{\phi(d(\eta(\omega), \zeta(\omega))), \phi(0)\}
\end{aligned}
$$

Consider

$$
\max \{\phi(d(\eta(\omega), \zeta(\omega))), \phi(0)\}=\phi(d(\eta(\omega), \zeta(\omega))),
$$

Then from (33), we have

$$
\varphi(d(\eta(\omega), \zeta(\omega))) \leq \phi(d(\eta(\omega), \zeta(\omega))) .
$$

Since $(\phi, \varphi) \in \mathfrak{F}$, we infer from Remark 3 that $d(\eta(\omega), \zeta(\omega))=$ 0 and so $\eta(\omega)=\zeta(\omega)$.

If

$$
\max \{\phi(d(\eta(\omega), \zeta(\omega))), \phi(0)\}=\phi(0),
$$

Then from (33), we have

$$
\varphi(d(\eta(\omega), \zeta(\omega))) \leq \phi(0) .
$$

Since $(\phi, \varphi) \in \mathfrak{F}$, we infer from condition (a2) that $d(\eta(\omega), \zeta(\omega)) \leq 0$ and so $\eta(\omega)=\zeta(\omega)$.

Therefore, in both cases we proved that $\eta(\omega)=\zeta(\omega)$.

Case. $2 \eta(\omega)$ is not comparable to $\zeta(\omega)$. Then there exists a measurable mapping $\xi(\omega): \Omega \rightarrow X$, which is comparable with both $\eta(\omega)$ and $\zeta(\omega)$. Now, we can define the sequence $\left\{\xi_{n}(\omega)\right\}$ from $\Omega$ to $X$ as follows :

$\xi_{0}(\omega)=\xi(\omega), \xi_{n+1}(\omega)=f\left(\omega, \xi_{n}(\omega)\right), \omega \in \Omega, n=0,1,2, \ldots$ where $\xi_{0}(\omega): \Omega \rightarrow X$ be an arbitrary measurable mapping.

Since $f$ is non-decreasing we have,

$$
\xi_{0}(\omega) \preceq \xi_{n}(\omega) \preceq \xi_{n+1}(\omega)
$$

Since $\xi_{n}(\omega)$ and $\xi_{n+1}(\omega)$ are comparable, applying (32), we can easily show that $\left\{d\left(\xi_{n+1}(\omega), \xi_{n}(\omega)\right)\right\}$ is a non-decreasing sequence such that

$$
\lim _{n \rightarrow \infty} d\left(\xi_{n+1}(\omega), \xi_{n}(\omega)\right)=0, \omega \in \Omega \text {. }
$$

As $\eta(\omega) \leq \xi_{n}(\omega)$, putting $x=\eta(\omega)$ and $y=\xi_{n}(\omega)$ in (32), we get

$$
\begin{aligned}
\varphi( & \left.d\left(\omega(\omega), \xi_{n+1}(\omega)\right)\right) \\
& =\varphi\left(d\left(f(\omega, \eta(\omega)), f\left(\omega, \xi_{n}(\omega)\right)\right)\right) \\
& \leq \max \left\{\phi\left(d\left(\eta(\omega), \xi_{n}(\omega)\right)\right),\right. \\
& \left.\phi\left(\frac{d\left(\xi_{n}(\omega), f\left(\omega, \xi_{n}(\omega)\right)\right)[1+d(\eta(\omega), f(\omega, \eta(\omega)))]}{1+d\left(\eta(\omega), \xi_{n}(\omega)\right)}\right)\right\} \\
& =\max \left\{\phi\left(d\left(\eta(\omega), \xi_{n}(\omega)\right)\right), \phi\left(\frac{d\left(\xi_{n}(\omega), f\left(\omega, \xi_{n}(\omega)\right)\right)}{1+d\left(\eta(\omega), \xi_{n}(\omega)\right)}\right)\right\}
\end{aligned}
$$

Let us denote

$$
\begin{aligned}
& G=\left\{n \in \mathbb{N}: \varphi\left(d\left(\eta(\omega), \xi_{n+1}(\omega)\right)\right) \leq \phi\left(d\left(\eta(\omega), \xi_{n}(\omega)\right)\right)\right\} \\
& H=\left\{n \in \mathbb{N}: \varphi\left(d\left(\eta(\omega), \xi_{n+1}(\omega)\right)\right) \leq \phi\left(\frac{d\left(\xi_{n}(\omega), f\left(\omega, \xi_{n}(\omega)\right)\right)}{1+d\left(\eta(\omega), \xi_{n}(\omega)\right)}\right)\right\}
\end{aligned}
$$

From (35), we have $\operatorname{Card} G=\infty$ or $\operatorname{CardH}=\infty$. Let us supposethat $\operatorname{Card} G=\infty$. Then there exists infinitely many $n \in \mathbb{N}$ satisfying

$$
\varphi\left(d\left(\eta(\omega), \xi_{n+1}(\omega)\right)\right) \leq \phi\left(d\left(\eta(\omega), \xi_{n}(\omega)\right)\right) .
$$

Since $(\varphi, \phi) \in \mathfrak{F}$, it follows that the sequence $\left\{d\left(\eta(\omega), \xi_{n}(\omega)\right)\right\}$ is non-increasing and it has a limit $l(\omega) \geq 0, \omega \in \Omega$. Since

$$
\begin{aligned}
\lim _{n \rightarrow \infty} d\left(\eta(\omega), \xi_{n+1}(\omega)\right) & \\
\quad & =\lim _{n \rightarrow \infty} d\left(\eta(\omega), \xi_{n}(\omega)\right)=l(\omega)
\end{aligned}
$$

We infer from (36) and condition (a3) that $l(\omega)=0, \omega \in \Omega$ and consequently, $\lim _{n \rightarrow \infty} \xi_{n+1}(\omega)=\eta(\omega), \omega \in \Omega$.
On the other hand, if $\operatorname{Card} H=\infty$, then we can find infinitely many $i \in \mathbb{N}$ satisfying

$$
\varphi\left(d\left(\eta(\omega), \xi_{n+1}(\omega)\right)\right) \leq \phi\left(\frac{d\left(\xi_{n}(\omega), f\left(\omega, \xi_{n}(\omega)\right)\right)}{1+d\left(\eta(\omega), \xi_{n}(\omega)\right)}\right)
$$

And since $(\varphi, \phi) \in \mathfrak{F}$, we obtain

$$
d\left(\eta(\omega), \xi_{n+1}(\omega)\right) \leq \frac{d\left(\xi_{n}(\omega), f\left(\omega, \xi_{n}(\omega)\right)\right)}{1+d\left(\eta(\omega), \xi_{n}(\omega)\right)}
$$

Taking the limit as $n \rightarrow \infty$ in last inequality and using (34), we obtain $\lim _{n \rightarrow \infty} d\left(\eta(\omega), \xi_{n+1}(\omega)\right)=0, \omega \in \Omega$ and consequently $\lim _{n \rightarrow \infty} \xi_{n+1}(\omega)=\eta(\omega), \omega \in \Omega$.

Therefore, in both cases we obtain

$$
\lim _{n \rightarrow \infty} \xi_{n+1}(\omega)=\eta(\omega), \omega \in \Omega
$$

In the same way it can be deduced that

$$
\lim _{n \rightarrow \infty} \xi_{n+1}(\omega)=\zeta(\omega), \omega \in \Omega
$$

Therefore passing to the limit in

$$
d(\eta(\omega), \zeta(\omega)) \leq d\left(\eta(\omega), \xi_{n+1}(\omega)\right)+d\left(\xi_{n+1}(\omega), \zeta(\omega)\right)
$$

As $n \rightarrow \infty$, we obtain $d(\eta(\omega), \zeta(\omega))=0$. Hence $\eta(\omega)=\zeta(\omega)$.

That is, the fixed point is unique.

Remark 23 Result similar to Corollary 12-20 involving various iterates of mappings corresponding to Corollary $21 \mathrm{can}$ also be derived. Due to repetition, the details are avoided.

\section{Acknowledgement}

The authors express deep gratitude to the referee for his/her valuable comments and suggestions.

\section{Author Contributions}

All authors contributed equally and significantly to writing this paper. All authors read and approved the final manuscript.

\section{Conflicts of Interest}

The authors declare no conflict of interest.

\section{REFERENCES}

[1] Agarwal, RP, Karapinar, E, \& Roldan-Lopez-de-Hierro, AF (2014), Fixed point theorems in quasi-metric spaces and applications, $\mathrm{J}$. Nonlinear Covex Anal.

[2] Altun, I, Simsek, H (2010), some fixed point theorems on ordered metric spaces and application, Fixed Point Theory and Applications, vol.2010, 1-17. Article ID 621469.

[3] Bergiz, M, Karapinar, E, Roldan, A (2014), Discussion on generalized- $(\alpha \psi, \beta \varphi)$-contractive mappings via generalized altering distance function and related fixed point theorems, Abstr. Appl. Anal., 2014, Article ID 259768.

[4] Cabrera, I, Harjani, J, Sadarangani, K (2013), A fixed point theorem for contractions of rational type in partially ordered metric spaces. Ann. Univ. Ferrara, 59, 251-258. http://dx.doi.org/10.1007/ s11565-013-0176-x.

[5] Chang, SS, Huang, NJ (1991), on the principle of randomization of fixed points for set valued mappings with applications, Northeastern Math. J., vol.7, 486-491.

[6] Dhage, BC (1999): Condensing mappings and applications to existence theorems for common solution of differential equations, Bull Korean Math. Soc., vol.36, no.3, 565-578.

[7] Hadzic, $\mathrm{O}(1979)$, A random fixed point theorem for multi valued mappings of Ciric's type. Mat. Vesnik 3 (16) (31), no. 4, 397-401.

[8] Hans, O (1957), Reduzierende Zufallige transformationen, Czech. Math. J. Vol.7, 154-158.

[9] Hans, O (1961), Random operator equations, Proc. 4th Berkeley Symp. Mathematics Statistics and Probability, Vol. II, Part I, pp. 185-202. University of California Press, Berkeley.

[10] Himmelberg, CJ (1975), Measurable relations. Fund. Math. Vol.87, 53-72.

[11] Huang, NJ (1999), a principle of randomization of coincidence points with applications, Applied Math. Lett. 12(1999), 107113.http://dx.doi.org/10.1016/S0893-9659(98)00157-8. 
[12] Itoh, S (1979): Random fixed-point theorems with an application to random differential equations in Banach spaces. J. Math. Anal. Appl. 67(2), 261-273.http://dx.doi.org/10.1016/0022-247X(79)900 23-4.

[13] Joshi, MC, Bose, RK(1984): Some Topics in Nonlinear Functional Analysis. Wiley, New York.

[14] Karapinar, E, Shatanawi, W, Tas, K (2013), Fixed point theorems on partial metric spaces involving rational expressions, Miskolc Math. Notes, 14, 135.142.

[15] Khan, MS, Swaleh, M, Sessa, S (1984), Fixed point theorems by altering distances between the points, Bull. Austr. Math. Soc., 30, 1-9.http://dx.doi.org/10.1017/S0004972700001659.

[16] Liu, TC (1988), Random approximations and random fixed points for non-self-maps, Proc. Amer. Math. Soc., vol.103, 11291135.http://dx.doi.org/10.1090/S0002-9939-1988-0954994-0.

[17] Moradi, S, Farajzadeh, A (2012), On the fixed point of $(\psi, \varphi)$-weak and generalized $(\psi, \varphi)$-weak contraction mappings, Appl. Math. Lett. $25,1257.1262$.

[18] Papageorgiou, NS(1984), Random fixed point theorems for multifunctions, Math. Japonica, vol. 29, 93-106.

[19] Papageorgiou, NS (1986), Random fixed point theorems for measurable multifunctions in Banach spaces, Proc. Amer. Math. Soc., vol.97, 507-514. http://dx.doi.org/10.1090/S0002-9939-1986-0840 638-3.

[20] Rocha, J, Rzepka, B, Sadarangani, K (2014), fixed point theorems for contraction of rational type with PPF dependence in Banach Spaces. Journal of Function Spaces, Article ID 416187, 18.http://dx.doi.org/10.1155/2014/416187.

[21] Rockafellar, RT (1969), Measurable dependence of convex sets and functions in parameters, J. Math. Anal. Appl., vol.28, 4-25. http://dx.doi.org/10.1016/0022-247X(69)90104-8.

[22] Saluja, AS, Khan, MS, Jhade, PK, Fisher, B (2015), some fixed point theorems for mappings involving rational type expressions in partial metric spaces. Applied Mathematics E-Notes, 15.

[23] Saluja, AS, Rashwan, RA, Magarde, D, Jhade, PK (2016), Some Result in Ordered Metric Spaces for Rational Type Expressions, Facta Universitatis, Ser. Math. Inform. Vol. 31, No 1, 125-138.

[24] Sehgal, VM, Singh, SP (1985), on random approximations and a random fixed point theorem for set valued mappings, Proc. Amer. Math. Soc., vol.95, 91-94.http://dx.doi.org/10.1090/S0002-99391985-0796453-1.

[25] Shahzad, N, Latif, A (2000), A random coincidence point theorem, J. Math. Anal. Appl., vol.245, 633-638.http://dx.doi.org/10.1006/ imaa.2000.6772.

[26] Spacek, a (1955), Zufallige Gleichungen, Czech Math. J., vol.5, 462-466.

[27] Tan, KK, Yuan, XZ, Huang, NJ (1994), Random fixed point theorems and approximations in cones, J. Math. Anal. Appl., vol.185, 378-390.http://dx.doi.org/10.1006/jmaa.1994.1256.

[28] Wagner DH (1977), Survey of measurable selection theorems, SIAM, J, Control Optim., vol.15, 859-903.http://dx.doi.org/10.1137 $\underline{10315056}$ 